\title{
Comparison of Two Surgical Approaches for Displaced Intra- Articular Calcaneal Fractures: The Modified Sinus Tarsi Approach Versus The Extensile Lateral Approach
}

\section{Chao Ma}

Second Affiliated Hospital of Soochow University

Jiaju Zhao

The Second Affiliated Hospital of Soochow University

Nan Yi

The Second Affiliated Hospital of Soochow University

Jupu Zhou

The Second Affiliated Hospital of Soochow University

\section{Zhicheng Zuo}

The Second Affiliated Hospital of Nanjing University of Chinese Medicine

Bo Jiang ( $\sim$ jiangbo082611@163.com )

The Second Affiliated Hospital of Nanjing University of Chinese Medicine

\section{Research article}

Keywords: Calcaneal fractures, Sinus tarsi approach, Extensile lateral approach, Minimally invasive

Posted Date: December 18th, 2020

DOI: https://doi.org/10.21203/rs.3.rs-129867/v1

License: (a) (1) This work is licensed under a Creative Commons Attribution 4.0 International License. Read Full License 


\section{Abstract}

Background: Displaced intra-articular calcaneal fractures (DIACF) can seriously affect foot function. The conventional surgical approach is the extensile lateral approach (ELA), but it has a high incidence of complications. To reduce the complications associated with ELA, many minimally invasive approaches have been suggested. The purpose of this study was to assess the clinical efficacy and complications of the ELA and a modified sinus tarsi approach (MSTA) for the treatment of DIACF.

Methods: One-hundred eight patients (107 feet) with Sanders II IV calcaneal fractures were retrospectively analysed, including 52 patients (56 feet) in the MSTA group and 56 patients (61 feet) in the ELA group. The functional and radiological results of the affected foot were analysed retrospectively. Functional evaluation included AOFAS, VAS, and SF-36. Radiological evaluation included preoperative and postoperative changes in the Bohler Angle, Gissane Angle, length, width, and height of the calcaneus. The postoperative complications were also collected and analysed.

Results: The average AOFAS scores of the MSTA group and the ELA group were $80.57 \pm 7.80$ and $77.34 \pm 8.29(p=0.033)$, respectively. The postoperative VAS scores of the MSTA group and the ELA group were $1.66 \pm 0.98$ and $2.03 \pm 0.98(p=0.043)$, respectively. The postoperative SF-36-PCS score of the MSTA group was superior to that of the ELA group $(77.91 \pm 5.03 \mathrm{vs.} 74.20 \pm 5.07, p<0.000)$, while there was no significant difference in the SF-36-MCS score ( $74.84 \pm 7.77$ vs $72.70 \pm 8.21, p=0.153)$. After surgery, the Bohler Angle, Gissane Angle, and the length, width, and height of the calcaneus were significantly improved in the MSTA group and the ELA group, but there were no statistically significant differences between the two groups. The incidence of wound healing complications in the MSTA group and the ELA group was $5.36 \%$ and $14.75 \%(p<0.000)$. Sural nerve injury and subtalar arthritis complications were less common in the MSTA group than in the ELA group $(p<0.000)$.

Conclusion: For DIACF, the MSTA was able to achieve similar effects of anatomical reconstruction and postoperative foot function recovery as the ELA, while also effectively reducing the incidence of postoperative sural nerve injury and wound healing complications and shortening the length of hospital stay.

\section{Introduction}

A calcaneal fracture is the most common fracture of the tarsal bones ${ }^{1}$. Approximately $75 \%$ of calcaneal fractures are intra-articular, which can seriously affect locomotion ${ }^{2}$. For displaced intra-articular calcaneal fractures (DIACF), open reduction and internal fixation (ORIF) is generally recommended ${ }^{3}$. Conservative treatment may cause malunion, subtalar arthritis, subfibular impingement and other sequelae, resulting in dysfunction or disability ${ }^{4}$. The primary goal of ORIF for DIACF is to restore the congruence of the subtalar joint. This fixation requires not only the anatomical reduction of the calcaneus, subtalar articular surface, and heel tubercle but also stable and firm internal fixator support to facilitate early rehabilitation exercise ${ }^{5}$.

The extensile lateral approach (ELA) has become a routine surgical approach for the treatment of DIACF, which can fully expose the location of the fracture and permit direct joint reduction. However, this approach can have multiple obvious complications, including superficial or deep infection, wound-edge necrosis, sural nerve injury, peroneal tendon injury, and subtalar arthritis ${ }^{6,7}$. To reduce the iatrogenic damage and wound complications caused by the ELA, many scholars have tried to use various minimally invasive approaches to treat intra-articular calcaneal fractures. The sinus tarsi approach (STA) is a frequently used method that is a minimally invasive approach. The STA and its modified schemes allow the surgeon to reduce the subtalar joint surface under direct vision and expose the calcaneal joint by extending the incision if necessary. In addition, locking plates can be inserted through these approaches to give the affected limb a firm fixation and minor soft tissue damage. The purpose of this study was to evaluate the clinical efficacy and complications of intra-articular calcaneal fractures after ORIF through a modified sinus tarsi approach (MSTA) and ELA.

\section{Material And Methods}

\section{Patients}

We retrospectively studied all of the patients admitted to our hospital for the repair of intra-articular calcaneal fractures between July

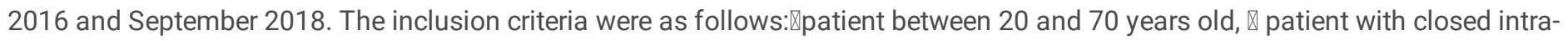
articular calcaneal fractures classified as Sander's type II IV, $\otimes$ all patients received ORIF via the MSTA or ELA, $\otimes$ patient had no major

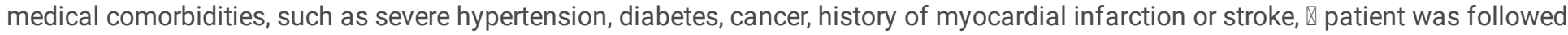


up for at least 12 months. The exclusion criteria were as follows: $\otimes$ patient suffered extra-articular or open calcaneal fractures, $₫$ other fresh fractures or neuromuscular injuries existed on the affected side of foot, $\otimes$ patient had rheumatoid arthritis or osteoarthritis, severe osteoporosis, severe peripheral vascular disease, and a history of calcaneal fractures. $\otimes$ patient was followed up for less than 12 months.

A total of 108 patients (117 feet) met these inclusion criteria and were included for analysis. The ethics committee of our hospital reviewed and approved this study. Fifty-two patients (56 feet) who were treated by the modified sinus tarsal approach were assigned to the MSTA group and 56 patients (61 feet) who were treated by the extensile lateral approach were assigned to the ELA group. All operations were performed by the same surgeon (Jiang Bo), and all of the patients were required to cease alcohol consumption and smoking after the calcaneal fractures.

There were 39 males $(75.00 \%)$ and 13 females $(25.00 \%)$ in the MSTA group, and their average age was $41.69 \pm 10.57$ years. According to the Sander's classification, there were 19 feet that were type II, 31 feet that were type $\varangle$, and 6 feet that were type $\otimes$. The time that had elapsed between injury and surgical intervention ranged from 2 to 8 days with an average of $4.11 \pm 1.27$ days. The total length of the hospital stay for patients was $8.23 \pm 1.73$ days. There were 43 males $(76.79 \%)$ and 13 females $(23.21 \%)$ in the ELA group and their average age was $44.95 \pm 10.62$ years. According to the Sander's classification, there were 19 feet that were type II, 37 feet that were type $\nabla$, and 5 feet that were type $\nabla$. The time that had elapsed between injury and surgical intervention ranged from 5 to 12 days with an average of $7.05 \pm 1.45$ days. The total length of the hospital stay for patients was $12.66 \pm 2.26$ days. Additional demographic data were collected for both groups, including the injured side and the mechanism of injury (Table 1).

\section{Modified Sinus tarsi approach}

General anaesthesia or spinal anaesthesia was administered in all of the patients. While under anaesthesia, the patient was placed in the lateral position with the affected side on the upper side, and a pneumatic tourniquet was applied to the thigh of affected side. The prone position was used if the patient had a bilateral calcaneal fracture. An incision was made from the tip of the lateral malleolus to the point of the anterior calcaneal process. The direction of the incision was parallel to the plane of the plantar. The main sural nerve was located in the area below the incision and did not need to be exposed during the dissection. The skin, subcutaneous tissue, and inferior extensor retinaculum were cut along the skin incision to expose the peroneal tendon sheath which was pulled downward. It was important to maintain the integrity of the tendon sheath and surrounding subcutaneous tissue. The extensor digitorum brevis terminal tendon was carefully stripped off and the sinus tarsi was exposed. The subtalar joint capsule was cut open to explore the posterior articular surface of the calcaneus, and the haematoma and adipose tissue were cleaned. The lateral calcaneal wall was stripped by sharp dissection through the sinus tarsi incision and the calcaneus ligament was cut off. Then, it was possible to expose and further reduce the collapsed posterior articular surface of the calcaneus using a periosteum stripper. A Kirschner wire was drilled below the articular surface along the direction of the sustentaculum tali for temporary fixation. A percutaneous Steinmann pin was drilled into the calcaneal tuberosity, which was used to reduce the varus deformity of the calcaneus and the comminuted lateral wall. The percutaneous Kirschner wires were then drilled along the long axis of the calcaneus for temporary fixation. Next, the lateral and axial positions of the calcaneus were observed by C-arm fluoroscopy to ensure that the length, width, and height of the calcaneus, the posterior articular surface, the Bohler Angle and the Gissane Angle were satisfactorily restored. Finally, a locking plate was inserted and placed on the lateral side of the calcaneus through the modified sinus tarsi incision. Several small incisions were made on the lateral calcaneal skin according to the location of the screw holes, through which the screws were inserted (Figure 1). In this group, no ligamentum calcaneus was repaired, and no bone grafting was performed on the bone defect. The incision was sutured after placing a negative pressure drainage ball or drainage strip. The affected limb was fixed with plaster or braces for 2-3 weeks after the operation, and postoperative weight-bearing began at the eighth week.

\section{Extensile lateral approach}

In this group, the standard ELA was used for reduction and fixation of the calcaneal fractures. First, a smooth L-shaped incision was made from between the lateral malleolus and the Achilles tendon to the bottom of the fifth metatarsal. The full-thickness flap which contained the sural nerve and peroneal tendons was separated at the periosteum level and fixed temporarily with three Kirschner wires. Next, the collapsed articular surface was pried up and the articular fragments were fixed with Kirschner wires. The calcaneus was restored to its normal shape by extruding and pulling. After the length, width, and height of calcaneus, the posterior articular surface, the Bohler Angle and the Gissane Angle were satisfactorily restored, a calcaneal locking plate was used for fixation (Figure 2). Postoperative management was the same as in the MSTA group described above. 


\section{Functional and radiological evaluation}

All patients underwent pre- and postoperative clinical and radiological evaluations. The results of the clinical evaluation were obtained from the American Orthopaedic Foot and Ankle Society (AOFAS) and included hindfoot score, the visual analogue scale (VAS), and the Short Form-36 Health Survey (SF-36) questionnaire. At the same time, we collected the postoperative complications experienced by all of the patients. The AOFAS score assigns 100 points to normal hindfoot and ankle joints, including pain (50 points), function (40 points) and alignment (10 points). We set a score of 90-100 as excellent, 75-89 as good, 50-74 as medium, and below 50 as poor. The VAS, which ranges from 0 (no pain) to 10 (extreme pain, seriously affects work and life), was used to measure the amount of pain. The SF-36 is a questionnaire that contains 36 items grouped into eight domains as follows: physical function, role physical, bodily pain, general health, vitality, social function, role emotional and mental health. Based on all of the items, a physical component summary (PCS) and a mental component summary (MCS) of health can be calculated. The SF-36-PCS comprises PF, RP, BP and GH. The SF-36MCS comprises VT, SF, RE and MH.

Radiological assessment indicators include the following aspects. Preoperative lateral and axial X-ray imaging and a CT scan were performed on the damaged calcaneus to evaluate the condition and classify the fractures. Lateral and axial X-ray radiographs of the damaged calcaneus were performed at 4 weeks, 3 months and 12 months after the operation. Then, the preoperative and postoperative changes of the Bohler Angle, Gissane Angle, calcaneal length, calcaneal width, and calcaneal height were calculated based on the X-ray imaging and CT scan results (Figure 3.4). Meanwhile, the healing of the calcaneal fractures and the incidence of complications were evaluated.

\section{Statistical Analysis}

Statistical analyses were performed using SPSS software (version 20.0). In this study, continuous variables were expressed as the means \pm standard deviations (SDs) and categorical variables were expressed as numbers $(\mathrm{N})$ and percentages (\%). The independent sample t-test and ANOVA were employed to compare differences between the two groups. A paired Student's t-test was performed to compare differences within the same group, and categorical variables were compared by a chi-square test. $P$ values $<0.05$ were considered statistically significant.

\section{Results}

The follow-up period of the MSTA group was 12 to 24 months, with an average of $16.65 \pm 2.74$ months, and the follow-up period for the ELA group was 12 to 30 months, with an average of $17.63 \pm 3.22$ months. After the last follow-up, the AOFAS score of the MSTA group was 67 99, with an average score of $80.57 \pm 7.80$, and the AOFAS score of the ELA group was 53 99, with an average score of $77.34 \pm 8.29(p=0.033)$. The favourable outcome rate of the affected feet was $80.36 \%$ in the MSTA group and $72.13 \%$ in the ELA group. The preoperative mean VAS score was $6.80 \pm 1.30$ for MSTA group and $6.79 \pm 1.18$ for ELA group $(p=0.942)$ and the last mean VAS score of the follow-up was $1.66 \pm 0.98$ for the MSTA group and $2.03 \pm 0.98$ for the ELA group $(p=0.043)$. The results of the SF-36 showed that the average PCS scores of the MSTA group and the ELA group were $77.91 \pm 5.03$ and $74.20 \pm 5.07(p<0.000)$, respectively and the average MCS score of the MSTA group and the ELA group was $74.84 \pm 7.77$ and $72.70 \pm 8.21 \quad(p=0.153$ ), respectively (Table 2).

Compared with the preoperative results, the lateral and axial calcaneal radiographs in the two groups showed good fracture reduction and fixation, and the postoperative Bohler Angle, Gissane Angle, calcaneal width, length, and height were significantly corrected $(p<0.000)$. However, no significant intergroup differences were observed for these variables $(p>0.05)($ Table 3$)$.

After the operation, the incidence of wound healing complications in the MSTA group and the ELA group was 5.36\% and $14.75 \%$ $(P<0.000)$, respectively. Three feet in the MSTA group had superficial infection of the surgical incision, which healed after dressing change. In the ELA group, five feet had superficial infection of the surgical incision and two feet had deep soft tissue infection with skin necrosis at the wound edge, which were cured after local debridement and dressing change. During the follow-up period, one foot suffered from sural nerve injury and dorsolateral foot numbness in the MSTA group. The symptoms were relieved one year after the operation without special treatment. In the ELA group, sural nerve injury occurred in four feet, and numbness on the dorsolateral side of the foot was obvious. Among them, three feet had reduced symptoms one year after the operation, while one foot had no significant changes. Two feet in the ELA group developed subtalar arthritis and tolerable pain, and received no special treatment during the follow-up period. There was no calcaneal malunion in either of the two groups. The incidence of sural nerve injury and subtalar arthritis in the MSTA group was lower than in the ELA group (Table 4). 


\section{Discussion}

A minimally invasive incision approach is considered the future direction in the development of calcaneal fracture treatment ${ }^{8}$. The traditional L-shaped incision cuts off the blood supply to the centre and front of the lateral wall of the calcaneus and destroys the vascular network of the plantar flap. Therefore, the complication rate of wound healing is $11 \% \sim 24.9 \%$, which seriously affects the treatment of calcaneal fractures ${ }^{6,9}$. Because of its minimally invasive nature, the sinus tarsi approach has been used more and more frequently in the treatment of calcaneal fractures. However, the traditional sinus tarsi approach runs diagonally downward from the tip of the lateral malleolus to the base of the fourth metatarsal bone, crossing the peroneal tendons, the main sural nerve and its anastomotic branches. Because of this, a 3-5\% chance of sural nerve damage still exists in the treatment of calcaneal fractures ${ }^{10-13}$. After anatomical study, Smyth NA ${ }^{10}$ believed that the traditional sinus tarsi approach would inevitably cause sural nerve injury. On the one hand, the trunk of the sural nerve is located in the deep part of the traditional tarsal sinus incision, leading to a high probability of iatrogenic injury. On the other hand, the main sural nerve above the incision may also produce branches, which may be injured and cause clinical symptoms during the surgical procedure.

Therefore, in order to minimize sural nerve injury and wound complications, we chose a modified tarsal sinus incision approach. The incision runs from the tip of the lateral malleolus to the apex of the anterior process of the calcaneus and is basically parallel to the plantar plane. Both the peroneal tendons and the main sural nerve are located in the area below the incision. During the operation, there is no need to expose the peroneal tendons and the sural nerve, and only the surface of the calcaneal lateral wall is needed for dissection. After the locking plate was well placed, several small skin incisions were made at the positions corresponding to the screw holes. Then, the incisions were expanded from the inside to the outside with vascular forceps by blunt separation, which allows the fixation of locking screws without damaging the sural nerve branch and subcutaneous small blood vessels as much as possible. This made it noteworthy that the length of the incision in the MSTA group was only $3-4 \mathrm{~cm}$. At the same time, we found that the postoperative blood loss of patients in the ELA group was much higher than the MSTA group. Some patients in the MSTA group did not have drainage tubes placed after surgery, and no obvious wound complications were found in postoperative follow-up. This showed that this minimally invasive technique had obvious advantages in reducing postoperative bleeding.

In this study, the rate of wound complications in the ELA group was $14.75 \%$, which was significantly higher than the MSTA group $(5.36 \%)$ and similar to relevant reports ${ }^{14}$. In terms of sural nerve injury, four dorsolateral feet (6.56\%) in the ELA group experienced numbness, while only one in the MSTA group (1.79\%) did. Among them, three feet in the ELA group and one foot in the MSTA group experienced spontaneous remission one year after surgery. We considered that these cases were related to excessive pulling of the skin and soft tissue and were not complications of sural nerve trunk injury. We believe that the advantages of the modified tarsal sinus incision are as follows. First, the exposure of the main sural nerve is avoided. Even if the branches of the main sural nerve which run upwards and distally are damaged in the incision area, there is still cross-innervation from the superficial peroneal nerve in this area, therefore, nerve injury symptoms would not be obvious. Second, the integrity of the peroneal tendon sheath and the subcutaneous tissue is preserved, which is convenient for the suture and repair of the subcutaneous tissue, and effectively reduces the damage to the plantar vascular network and the lateral posterior foot artery, and results in fewer postoperative incision complications. Third, the small incision can effectively reduce intraoperative and postoperative blood loss and promote wound healing.

In addition, another important finding of our study was that due to the protection of the soft tissue and blood supply, the wait time of patients in the MSTA group from injury to surgery was significantly shorter than in the ELA group $(4.11 \pm 1.27$ days vs. $7.05 \pm 1.45$ days, $\mathrm{P}<0.000)$. A study by Abidi ${ }^{15}$ et al. showed that the greater the number of days between injury and surgery, the higher the incidence of wound healing complications in DIACF. Their study found that in the ELA treatment, the incidence of wound complications was $16.6 \%$ among patients who received surgery 4.8 days after injury and $42.5 \%$ among patients who received surgery 9.9 days after injury. Similarly, Kwon ${ }^{16}$ et al. concluded that the delayed fixation of closed DIACF was associated with an increased incidence of wound complications in the STA treatment. In their study, if the fixation of the fractures was delayed for more than two weeks, the incidence of wound complications significantly increased, up to $15.2 \%$, while the incidence of wound complications was only $2.1 \%$ among patients treated within one week of injury. However, Shaoliang $\mathrm{Li}^{17}$ held the opposite view, suggesting that a 6 -day soft tissue recovery period may be necessary for safety in the STA treatment, which would otherwise lead to a higher incidence of complications and severe wound complications. This study validates the views of Abidi and Kwon by indicating that patients in the STA group did not need to wait for limb swelling to completely disappear under the condition of similar injury severity and surgical technique. In our study, 13 feet (23.21\%) in the MSTA group underwent surgery while still having tension blisters, and no wound healing complications occurred after surgery. In contrast, patients in the ELA group had to wait for the swelling to subside and skin 
lines to appear in order to avoid as many surgical incision healing complications as possible. As a result, the overall length of hospital stay in the MSTA group was shorter than in the ELA group, which reduced the economic burden on the patients and ensured a favourable prognosis and satisfaction with the treatment.

According to the previous reports, it is worth noting that although the use of minimally invasive techniques can effectively reduce the occurrence of soft tissue complications, the sinus tarsi approach still suffers from poor visualization, inadequate reduction, and unreliable fixation when compared to the ELA ${ }^{18}$. More specifically, the reduction of incision-related complications requires minimal incisions, while intra-articular fractures require good anatomic reduction ${ }^{4,19}$. The fragility of the calcaneus requires strong internal fixation, especially in the treatment of older patients with comminuted calcaneal fractures, severely compressed articular surface calcaneal fractures, and osteoporotic fractures. However, we found that although intraoperative exposure was limited, we were able to directly reduce the posterior facet joints and place the calcaneal locking plate through the MSTA. Combined with percutaneous nailing, we were able to minimize the incision while providing more powerful internal fixation support than screws, Kirschner pins, or miniature steel plates. After surgery, the Bohler Angle, Gissane Angle, and the length, width, and height of the calcaneus were significantly improved in the MSTA group and the ELA group, and there were no statistically significant differences between the two groups. The VAS score and SF-36 score of the two groups also showed no significant difference, and the AOFAS hindfoot score of the MSTA group was higher than the ELA group. The results showed that the modified sinus tarsi approach combined with the locking plate internal fixation could achieve anatomical reconstruction of the calcaneus and was slightly superior to the traditional ELA approach in postoperative foot function recovery. Through summary analysis, we found that MSTA is a challenging technique that requires a thorough understanding of the fracture type and calcaneal anatomy, which requires a steep learning curve for young physicians. However, once mastered, this technique will effectively reduce the incidence of postoperative sural nerve injury and wound healing complications and shorten the length of hospital stay.

Our study suffered from several limitations. First, our study on severe complex calcaneal fractures was insufficient. Our study proved

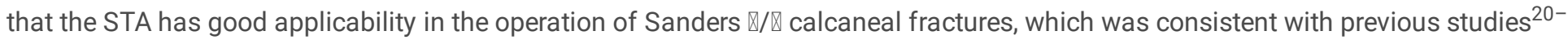

22. However, this study did not involve many cases of Sanders IV, senile and osteoporotic calcaneal fractures, and is therefore not representative of these fracture types. Second, the follow-up time was short, and this study lacked a long-term efficacy evaluation and randomized controlled study design. Therefore, it is necessary to conduct further studies with a larger sample size, longer follow-up time, and randomized controlled trials to obtain more accurate efficacy evaluation.

\section{Conclusion}

In summary, our study showed that for DIACF, the MSTA was able to achieve similar effects of anatomical reconstruction and postoperative foot function recovery as the ELA, while effectively reducing the incidence of postoperative sural nerve injury and wound healing complications and shortening the length of hospital stay. Therefore, the MSTA has become our preferred method for the treatment of DIACF in clinical practice.

\section{Abbreviations}

AOFAS American Orthopaedic Foot and Ankle Society

DIACF Displaced intra-articular calcaneal fractures

ELA Extensile lateral approach

MCS Mental component summary

MSTA Modified sinus tarsi approach

ORIF Open reduction and internal fixation

PCS Physical component summary

SF-36 Short Form-36 Health Survey

STA Sinus tarsi approach 


\section{Declarations}

\section{Ethics approval and consent to participate}

The study was reviewed and approved by the Ethics Committee of the Second Affiliated Hospital of Soochow University.

\section{Consent for publication}

\section{All patient's data involved in this study were agreed for publication.}

\section{Availability of data and materials}

\section{The datasets used and/or analysed during the current study are available from the corresponding author on reasonable request.}

\section{Competing interests}

\section{The authors declare that they have no competing interests}

\section{Funding}

(1) The Program of Key Research and Development of Jiangsu Province (Grant No. BE2018656)凶

(2) The Key Medical Discipline in Suzhou (Grant No. Szxk201802),

(3) Suzhou Key Laboratory of Peripheral Nerve Injury Repair (Grant No. SZS201720),

(4) Suzhou Clinical Key Disease Diagnosis and Treatment Technology Foundation (Grant No. LCZX201708),

(5) Training Project of 'National Tutor System' for Young Health Talents in Suzhou and the Science and Technology and Planning Program of Suzhou (Grant No. SZS2019063).

\section{Authors' contributions}

BJ designed this research. NY, JPZ and ZCZ participated in data collection. CM and JJZ participated in analysis and interpretation of data and revised the draft. CM and JJZ were two major contributors contributed equally to the writing of this manuscript. All authors read and approved the final manuscript.

\section{Acknowledgements}

We would like to acknowledge financial support from the Program of Key Research and Development of Jiangsu Province (Grant No. BE2018656), Key Medical Discipline in Suzhou (Grant No. Szxk201802), Suzhou Key Laboratory of Peripheral Nerve Injury Repair (Grant No. SZS201720), Suzhou Clinical Key Disease Diagnosis and Treatment Technology Foundation (Grant No. LCZX201708), Training Project of 'National Tutor System' for Young Health Talents in Suzhou and the Science and Technology and Planning Program of Suzhou (Grant No. SZS2019063).

\section{References}

1. Buzzi R, Sermi N, Soviero F, Bianco S, Campanacci DA. Displaced intra-articular fractures of the calcaneus: ORIF through an extended lateral approach. Injury. 2019:S2-S7.

2. Bai L, Hou YL, Lin GH, Zhang X, Liu GQ, Yu B. Sinus tarsi approach (STA) versus extensile lateral approach (ELA) for treatment of closed displaced intra-articular calcaneal fractures (DIACF): A meta-analysis. Orthopaedics \& traumatology, surgery \& research : OTSR. 2018;104(2):239-244.

3. Veltman ES, Doornberg JN, Stufkens SA, Luitse JS, van den Bekerom MP. Long-term outcomes of 1,730 calcaneal fractures: systematic review of the literature. The Journal of foot and ankle surgery : official publication of the American College of Foot and Ankle Surgeons. 2013;52(4):486-490. 
4. Pitts CC, Almaguer A, Wilson JT, Quade JH, Johnson MD. Radiographic and Postoperative Outcomes of Plate Versus Screw Constructs in Open Reduction and Internal Fixation of Calcaneus Fractures via the Sinus Tarsi. Foot \& ankle international. 2019;40(8):929-935.

5. Xu J, He Z, Zhang G, et al. An experimental study on the digital precision of internal fixation via the sinus tarsi approach for calcaneal fractures. Journal of orthopaedic surgery (Hong Kong). 2019;27(1):2309499019834072.

6. Zhan J, Hu C, Zhu N, Fang W, Jing J, Wang G. A modified tarsal sinus approach for intra-articular calcaneal fractures. Journal of orthopaedic surgery (Hong Kong). 2019;27(2):2309499019836165.

7. Seat A, Seat C. Lateral Extensile Approach Versus Minimal Incision Approach for Open Reduction and Internal Fixation of Displaced Intra-articular Calcaneal Fractures: A Meta-analysis. The Journal of foot and ankle surgery : official publication of the American College of Foot and Ankle Surgeons. 2020;59(2):356-366.

8. Carr JB. Surgical treatment of intra-articular calcaneal fractures: a review of small incision approaches. Journal of orthopaedic trauma. 2005;19(2):109-117.

9. Nosewicz TL, Dingemans SA, Backes M, Luitse JSK, Goslings JC, Schepers T. A systematic review and meta-analysis of the sinus tarsi and extended lateral approach in the operative treatment of displaced intra-articular calcaneal fractures. Foot and ankle surgery : official journal of the European Society of Foot and Ankle Surgeons. 2019;25(5):580-588.

10. Smyth NA, Zachwieja EC, Buller LT, Miranda AD, Steinlauf SD. Surgical approaches to the calcaneus and the sural nerve: There is no safe zone. Foot and ankle surgery : official journal of the European Society of Foot and Ankle Surgeons. 2018;24(6):517-520.

11. Kline AJ, Anderson RB, Davis WH, Jones CP, Cohen BE. Minimally invasive technique versus an extensile lateral approach for intraarticular calcaneal fractures. Foot \& ankle international. 2013;34(6):773-780.

12. Yeo JH, Cho HJ, Lee KB. Comparison of two surgical approaches for displaced intra-articular calcaneal fractures: sinus tarsi versus extensile lateral approach. BMC musculoskeletal disorders. 2015;16:63.

13. Zhang T, Su Y, Chen W, Zhang Q, Wu Z, Zhang Y. Displaced intra-articular calcaneal fractures treated in a minimally invasive fashion: longitudinal approach versus sinus tarsi approach. The Journal of bone and joint surgery. American volume. 2014;96(4):302-309.

14. Daws SB, Neary K, Lundeen G. Short-Term Radiographic Outcomes of Calcaneus Fractures Treated With 2-Incision, Minimally Invasive Approach. Foot \& ankle international. 2019;40(9):1060-1067.

15. Shuler FD, Conti SF, Gruen GS, Abidi NA. Wound-healing risk factors after open reduction and internal fixation of calcaneal fractures: does correction of Bohler's angle alter outcomes? The Orthopedic clinics of North America. 2001;32(1):187-192, x.

16. Kwon JY, Guss D, Lin DE, et al. Effect of Delay to Definitive Surgical Fixation on Wound Complications in the Treatment of Closed, Intra-articular Calcaneus Fractures. Foot \& ankle international. 2015;36(5):508-517.

17. Li S. Wound and Sural Nerve Complications of the Sinus Tarsi Approach for Calcaneus Fractures. Foot \& ankle international. 2018;39(9):1106-1112.

18. Bedigrew KM, Blair JA, Possley DR, Kirk KL, Hsu JR. Comparison of Calcaneal Exposure Through the Extensile Lateral and Sinus Tarsi Approaches. Foot \& ankle specialist. 2018;11(2):142-147.

19. De Boer AS, Van Lieshout EM, Den Hartog D, Weerts B, Verhofstad MH, Schepers T. Functional outcome and patient satisfaction after displaced intra-articular calcaneal fractures: a comparison among open, percutaneous, and nonoperative treatment. The Journal of foot and ankle surgery : official publication of the American College of Foot and Ankle Surgeons. 2015;54(3):298-305.

20. Kikuchi C, Charlton TP, Thordarson DB. Limited sinus tarsi approach for intra-articular calcaneus fractures. Foot \& ankle international. 2013;34(12):1689-1694.

21. Basile A, Albo F, Via AG. Comparison Between Sinus Tarsi Approach and Extensile Lateral Approach for Treatment of Closed Displaced Intra-Articular Calcaneal Fractures: A Multicenter Prospective Study. The Journal of foot and ankle surgery : official publication of the American College of Foot and Ankle Surgeons. 2016;55(3):513-521.

22. Lin J., Xie C., Chen K., et al. Comparison of sinus tarsi approach versus extensile lateral approach for displaced intra-articular calcaneal fractures Sanders type IV. International orthopaedics. 2019;43(9):2141-2149.

\section{Tables}

Table 1 Demographic data of the patients 


\begin{tabular}{|llll|}
\hline & MSTA group & ELA group & p value \\
\hline Sex (male/female) & $39 / 13$ & $43 / 13$ & 0.828 \\
\hline Age (years, mean \pm SD) & $41.69 \pm 10.57$ & $44.95 \pm 10.62$ & 0.114 \\
\hline Side (Right foot/Left foot) & $30 / 26$ & $34 / 27$ & 0.814 \\
\hline Aetiology (n, percentage) & & & 0.639 \\
Fall from a height & $46(82.14 \%)$ & $48(78.69 \%)$ & \\
Traffic accidents & $10(17.86 \%)$ & $13(21.31 \%)$ & \\
Sanders classification (n, percentage) & & & 0.816 \\
Type II & $19(33.93 \%)$ & $19(31.15 \%)$ & \\
Type III & $31(55.36 \%)$ & $37(60.65 \%)$ & \\
Type IV & $6(10.71 \%)$ & $5(8.20 \%)$ & \\
\hline Wait time from injury to surgery (days, mean $\pm S D)$ & $4.11 \pm 1.27$ & $7.05 \pm 1.45$ & 0.000 \\
\hline Length of hospital stay (days, mean $\pm S D)$ & $8.23 \pm 1.73$ & $12.66 \pm 2.26$ & 0.000 \\
\hline
\end{tabular}

Table 2 Functional evaluation parameters of the two groups

\begin{tabular}{|llll|}
\hline & MSTA group & ELA group & p value \\
\hline Follow-up time (months, mean \pm SD) & $16.65 \pm 2.74$ & $17.63 \pm 3.22$ & 0.096 \\
\hline AOFAS (score,mean \pm SD) & $80.57 \pm 7.80$ & $77.34 \pm 8.29$ & 0.033 \\
\hline Excellent & $17.86 \%$ & $13.11 \%$ & - \\
\hline Good & $62.50 \%$ & $59.02 \%$ & - \\
\hline Fair & $19.64 \%$ & $27.87 \%$ & - \\
\hline Preoperative-VAS & $6.80 \pm 1.30$ & $6.79 \pm 1.18$ & 0.942 \\
\hline Postoperative-VAS & $1.66 \pm 0.98$ & $2.03 \pm 0.98$ & 0.043 \\
\hline SF-36-PCS & $77.91 \pm 5.03$ & $74.20 \pm 5.07$ & 0.000 \\
\hline SF-36-MCS & $74.84 \pm 7.77$ & $72.70 \pm 8.21$ & 0.153 \\
\hline
\end{tabular}

Table 3 Radiological evaluation parameters of the two groups 


\begin{tabular}{|c|c|c|c|c|c|c|c|c|}
\hline & \multicolumn{3}{|l|}{ MSTA group } & \multicolumn{3}{|l|}{ ELA group } & \multicolumn{2}{|l|}{ MSTA vs. ELA } \\
\hline & Preoperative & Postoperative & $\begin{array}{l}\text { Within } \\
\mathrm{p} \\
\text { value }\end{array}$ & Preoperative & Postoperative & $\begin{array}{l}\text { Within } \\
\mathrm{p} \\
\text { value }\end{array}$ & $\begin{array}{l}\mathrm{p} \text { value } \\
\text { preoperative }\end{array}$ & $\begin{array}{l}\mathrm{p} \text { value } \\
\text { postoperative }\end{array}$ \\
\hline $\begin{array}{l}\text { Bohler } \\
\text { angle }\end{array}$ & $13.07 \pm 7.87$ & $27.27 \pm 3.97$ & 0.000 & $11.20 \pm 7.13$ & $28.61 \pm 4.71$ & 0.000 & 0.179 & 0.101 \\
\hline $\begin{array}{l}\text { Gissane } \\
\text { angle }\end{array}$ & $118.16 \pm 113.23$ & $124.70 \pm 6.87$ & 0.000 & $117.85 \pm 12.97$ & $126.59 \pm 4.55$ & 0.000 & 0.899 & 0.079 \\
\hline Length & $71.11 \pm 3.88$ & $77.14 \pm 2.98$ & 0.000 & $71.38 \pm 3.00$ & $77.44 \pm 3.00$ & 0.000 & 0.589 & 0.766 \\
\hline Width & $54.07 \pm 5.20$ & $46.05 \pm 3.89$ & 0.000 & $55.33 \pm 5.86$ & $46.84 \pm 4.74$ & 0.000 & 0.333 & 0.386 \\
\hline Height & $39.73 \pm 3.22$ & $45.04 \pm 3.65$ & 0.000 & $38.54 \pm 3.10$ & $44.36 \pm 2.82$ & 0.000 & 0.044 & 0.263 \\
\hline
\end{tabular}

Table 4 Complications after surgery

\begin{tabular}{|llllll|}
\hline & \multicolumn{2}{l}{ MSTA group } & \multicolumn{2}{l}{ ELA group } & \multirow{2}{*}{ p value } \\
\cline { 2 - 5 } & number & proportion & number & proportion & \\
Wound healing complications & 3 & $5.36 \%$ & 9 & $14.75 \%$ & 0.000 \\
\hline Superficial infection & 3 & $5.36 \%$ & 5 & $8.20 \%$ & \\
\cline { 1 - 4 } Deep infection & 0 & 0 & 2 & $3.28 \%$ & \\
\hline Wound-edge necrosis & 0 & 0 & 2 & $3.28 \%$ & \\
\hline Sural nerve injury & 1 & $1.79 \%$ & 4 & $6.56 \%$ & 0.000 \\
\hline Subtalar arthritis & 1 & $1.79 \%$ & 2 & $3.28 \%$ & 0.000 \\
\hline
\end{tabular}

\section{Figures}




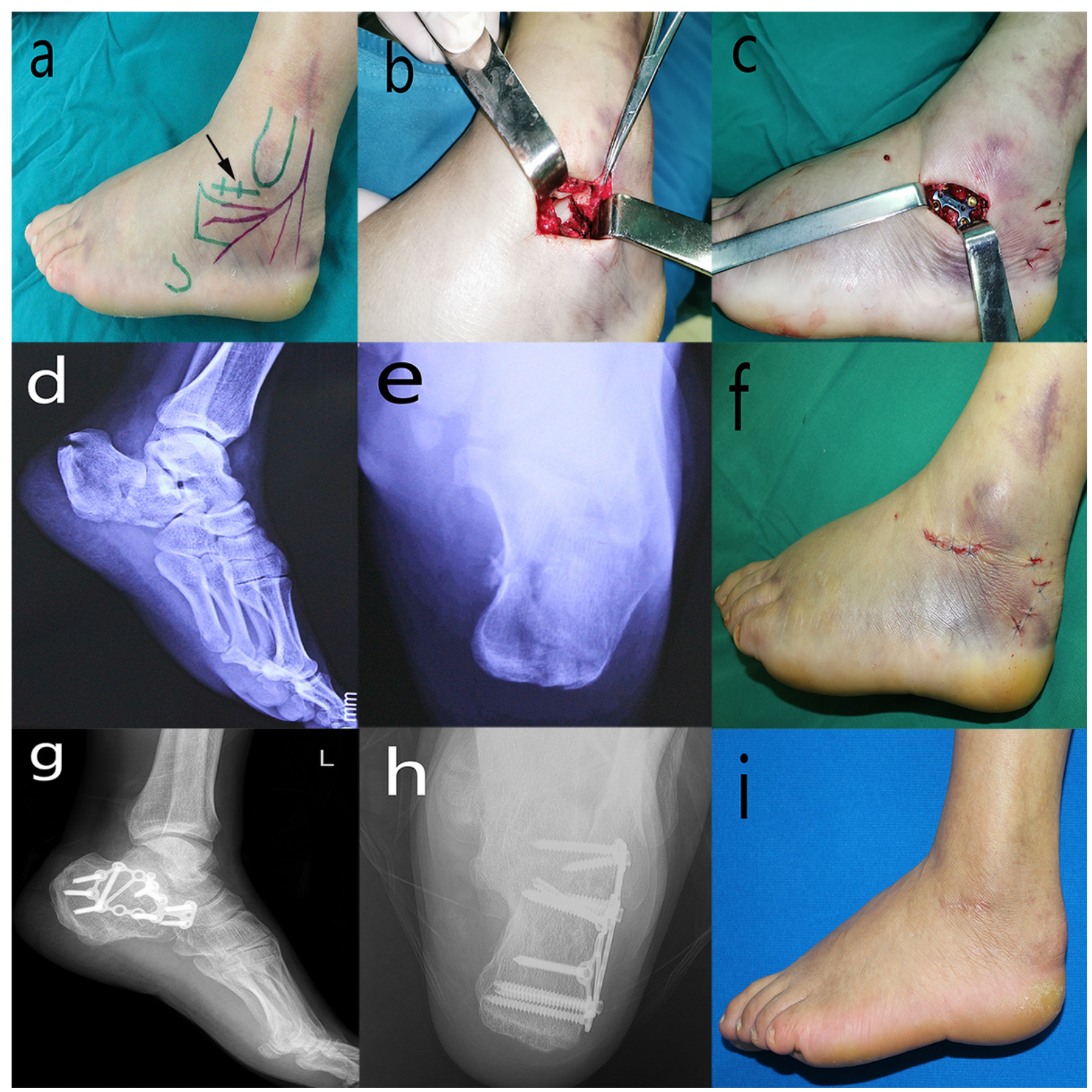

\section{Figure 1}

a The surgical incision marker (arrow) and sural nerve marker (purple lines) of MSTA. b The posterior articular surface of the calcaneus was exposed. c A locking plate was inserted and placed on the lateral side of the calcaneus. d,e Preoperative lateral and axial X-ray imaging. $f$ The MSTA incision was sutured. g,h Postoperative lateral and axial X-ray imaging. $i$ The appearance of the incision scar 12 months after surgery. 


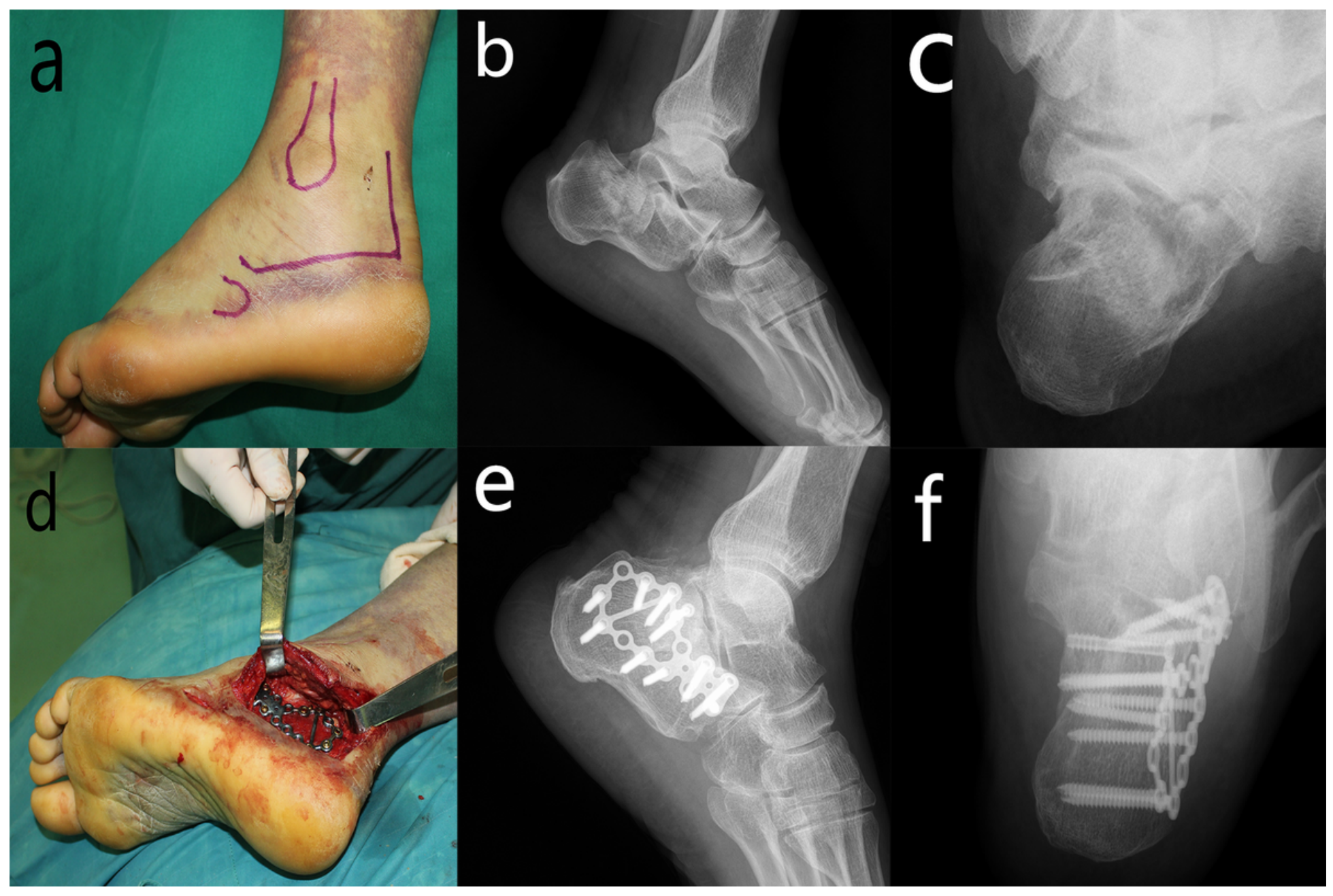

\section{Figure 2}

a The surgical incision marker of ELA. b,c Preoperative lateral and axial X-ray imaging. $d$ A locking plate was inserted and placed on the lateral side of the calcaneus. e,f Postoperative lateral and axial X-ray imaging. 


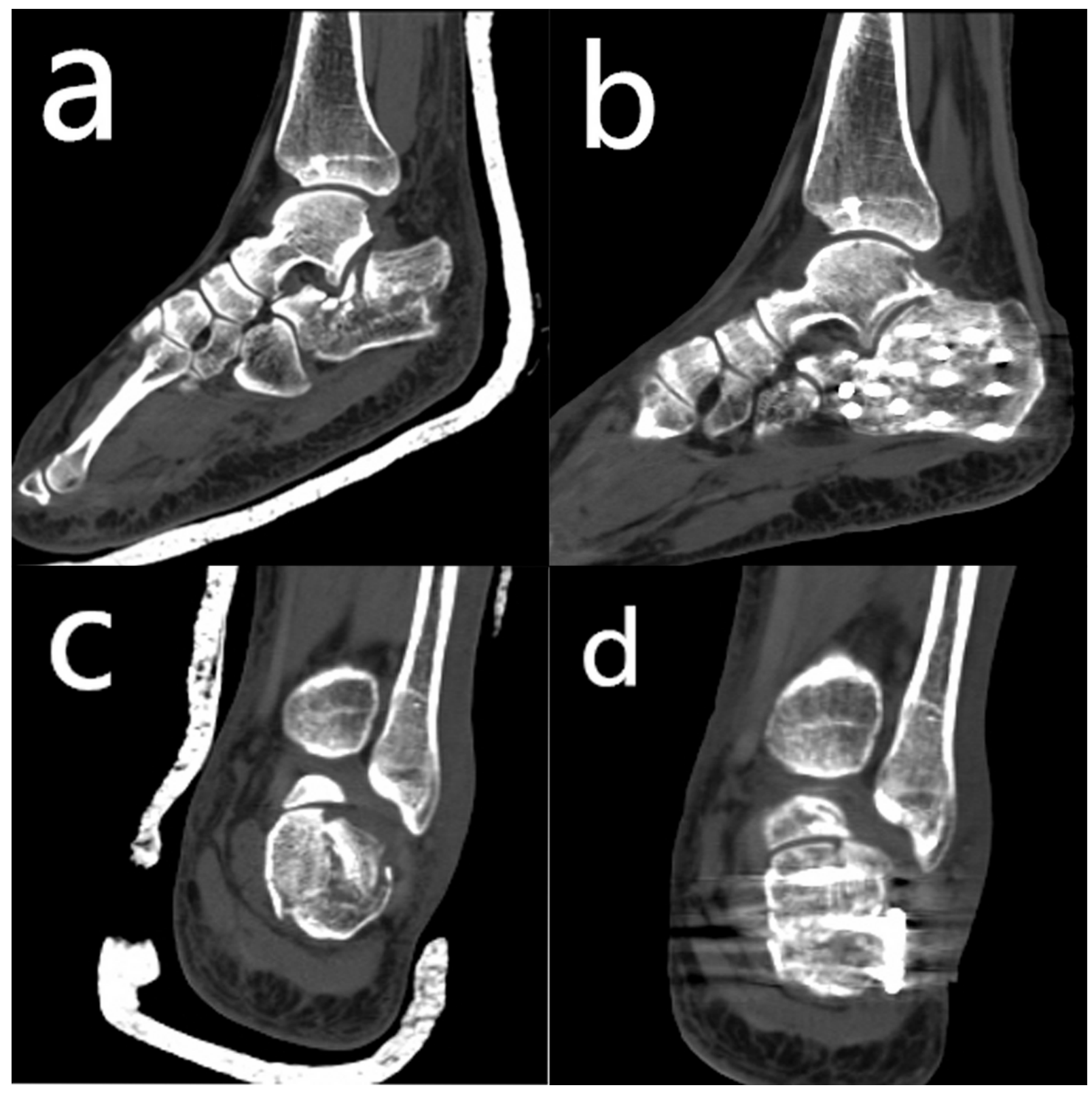

Figure 3

A patient treated through MSTA. a,b Preoperative and postoperative lateral CT scan imaging. c,d Preoperative and postoperative axial CT scan imaging. 


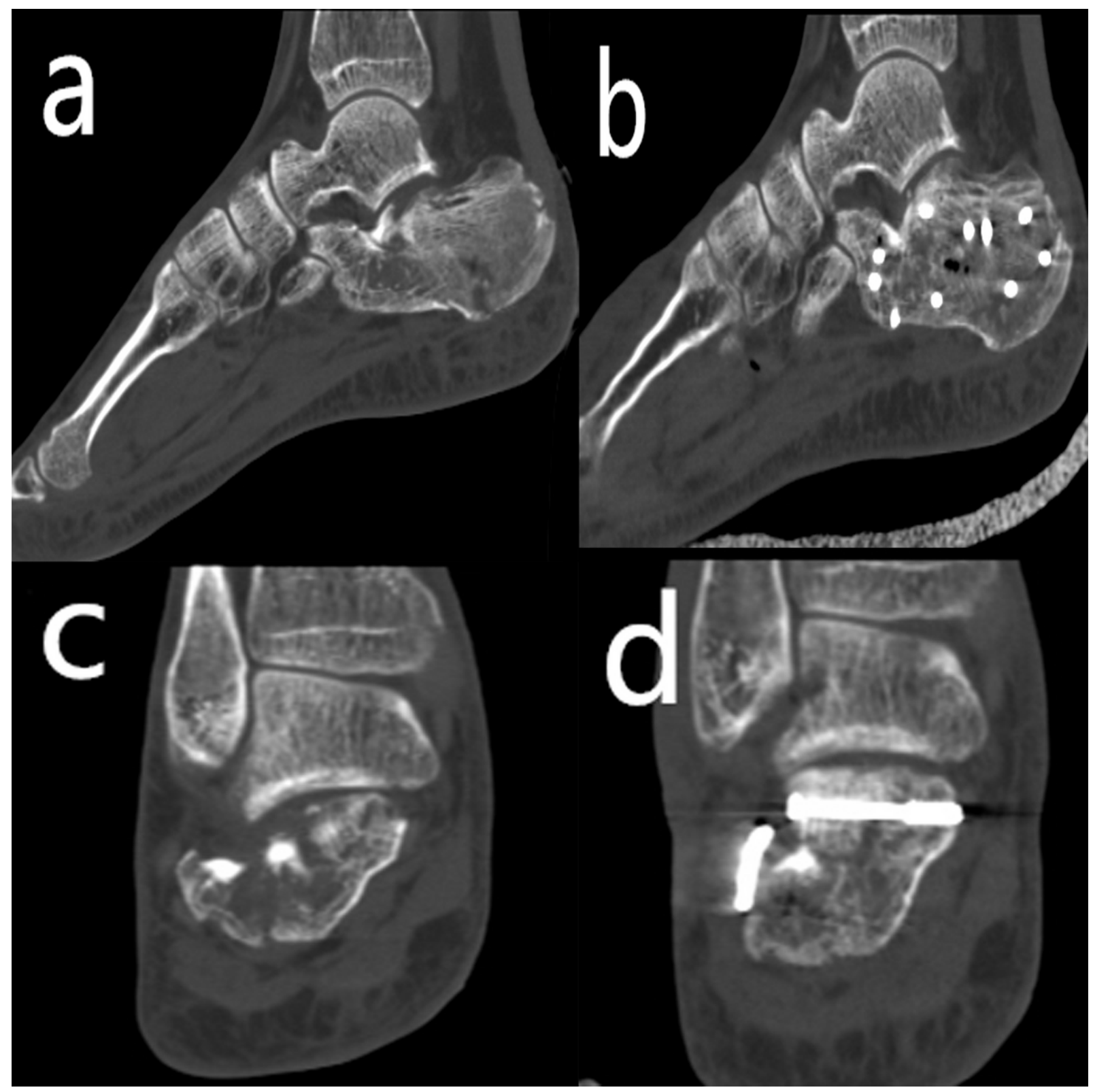

Figure 4

A patient treated through ELA. a,b Preoperative and postoperative lateral CT scan imaging. c,d Preoperative and postoperative axial CT scan imaging. 